noch keine Erwähnung dieses Tieres gefunden, und daher schien die Annahme, dass erst die Kassiten es nach Babylonien gebracht hätten, berechtigt. Unsere Urkunde indes, die ihrer Schrift und ihrem ganzen Aussehen nach etwa in die Zeit Hammurabis oder Samsuilunas gesetzt werden muss, zeigt, wie vorsichtig man mit Beweisen ex silentio sein muss: es ist gewiss ein reiner Zufall dass das Pferd sonst in altbabylonischen Urkunden nicht erwähnt wird. Allerdings spielen die Kassiten schon zur Zeit Samsuilunas eine Rolle1); doch sehe ich nicht ein, warum sie gerade den ,Bergesel" nach $\mathrm{Ba}$ bylonien gebracht haben sollten; er kann dorthin sehr gut schon viel früher durch Handelsbeziehungen mit den östlichen Völkerschaften gekommen sein.

Der Text des Briefes VAT 6088 lautet: V.AT. 6088.

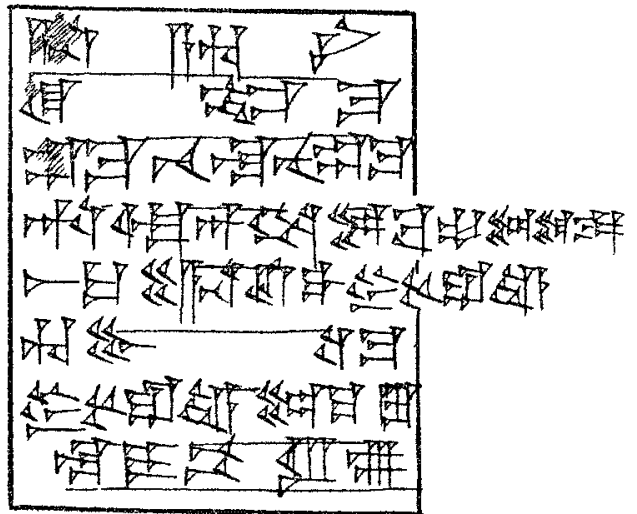

Rürkseite unbeschrioben.

${ }^{1}$ A-na A-hu-ni ${ }^{2}$ qi-bé-ma ${ }^{3}$ um-ma Be-lanu-um-ma 4 il Šamaš $\hat{u}$ il Marduk li-ba-al-li-tuka ${ }^{5}$ išten gur še'im a-na ukulli $\left.{ }^{2}\right)$ sisî zun ${ }^{6}$ bubu-ut-ma ${ }^{7}$ sisû zun li-ku-lu ${ }^{8}$ la i-bi-ru-u, d. i.

,Zu Ahuni sprich: also sagt Belanum: Šamaš und Marduk mögen Dich gesund erhalten! 1 Gur Getreide nimm fort als Futter für die Pferde, damit die Pferde zu fressen haben und nicht Hunger leiden müssen."
Berlin.
A. Ungnad.

Die von wir in ZDMG 61, 716 vor Erscheinen ron Kings Buch angenommenen Daten wären demnach um je 94 Jahre zu erhöhen.

1) Vgl. das Datum des neunten Jahres und daza BA VI 3, S. 29.

v) SAG. GAL.

\section{Assyrische Medizinalpflanzen.}

Von A. Eonahn.

Ich möchte - als Mediziner und nicht Philologe - fragen, ob folgende Zusammenstellungen von philologischem Gesichtspunkt aus zulässig sind und weitere Untersuchungen verdienen. Die Namen sind bei $K$ üchler: Assyr. Mediz. zu finden.

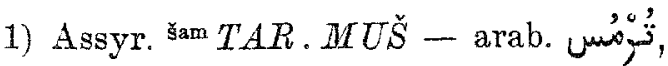

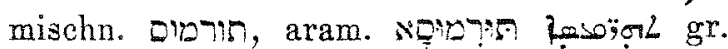
9 éouos (siehe Löw: Aram. Ptanzenn.) = Lupinus, Lupine.

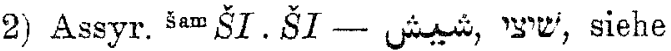
Löw: A. Pfl., Seite $114=$ peine Art schlechter Datteln". - Vielleicht wäre die Determinierung šammu hinderlich, obgleich dieses Wort ofters als ein weiterer Begriff als ${ }_{\text {Pflanze }}$ erscheint.

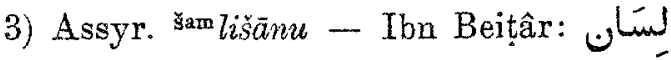
$=$ Erysimum, Hederich oder Schotendotter.

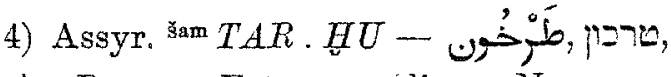

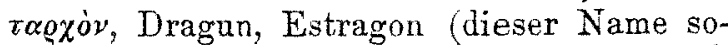
gar an der Westküste Norwegens!); auch im Keltischen. = Artemisia Dracunculus L.

5) Assyr. รัam $D I L . B A T(\operatorname{oder} B \hat{E})-$

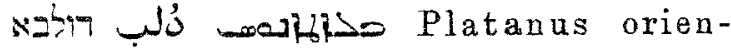
talis L. (Auch hier šammu!).

6) Assyr. šamkaran šêlibi "Fuchswein" -

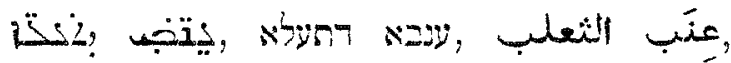

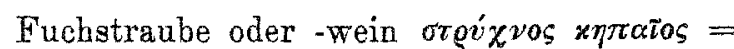
Solanum nigrum L.

7) Assyr. buttati - אavy = Frucht o(i)?

des Kappernstrauches, Capparis spinosa L.

8) Assyr. is $S I . B A$ (Küchler: K. $71 \mathrm{~b}$, IV 50) $=$ is $S I . H U$ (CT. Part XXIII, Serie: ênuma(?) amêlu muhbुi-šu išata u-kâl $P$ l.

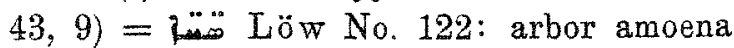
et infrugifera [Ferr].

9) Hängt giparu mit $\times=51=$, ijoa mandäisch Nosม zusammen? Bedeutung vielleicht eine Art Palme? (Talmudisch eig. Palmzweig; arab. Bezeichnungen für Teile der Palme stammen ja aus Babylon.

10) Assyr. rik (in CT. XXIII seria citat.: 


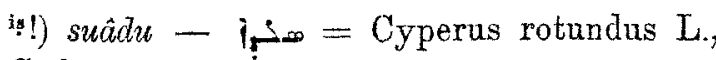
C. longus $\mathrm{I}$., Cyperngras.

11) Assyr. abukatu - smpes, Löw Seite 114: Seitenschösse, die die Palme zu Anfang treibt. - "פN stammt aus Babylon!

12) Assyr. rotes $S A . P A$ - has

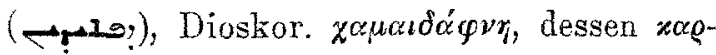
nov. .. É Ev $\vartheta$ oov $=$ Ruseus racemosus, Traubiger Mâusedorn (Sprengel). Plinius: Viniapervinea. - Dioskor: "Ihre Blätter, ... mit Wein getrunken, lindern das Leibschneiden, der Saft .... treibt den Harn".

13) Assyr. šam (oder is) hašu - Nert,

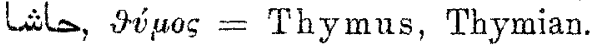

14) Schon bei Muss-Arnolt is dup-ra-nu

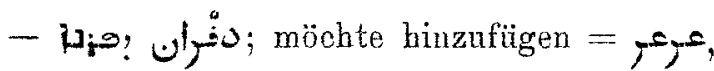
Juniperus, besonders J. sabina L., Sevenbaum, Sadebaum (siehe Löw).

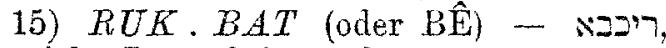
siehe Löw, Seite 120 .

\section{An hang.}

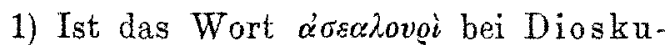
rides - nach ihm ägyptisch - - Pap. Ebers, 63, 9: - 0 ons 111 , cnfr. Stern: "वat'aulen' grana quaedam phoenicia “? Bei Löw ist dieses Wort unter seinen "punischen Pflanzennamen" nicht zu finden.

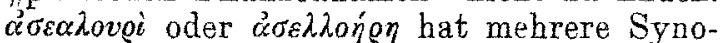
nymen bei den "Propheten und anderen" wie: Fuss des Hermes, Diadem des Osiris, Sonnenkrone, der heilige Stengel. Identifizierung: ¿2unog, Atriplex Halimus L., Meldenstrauch.

2) Löw: A. PAl fragt, Seite 313: Woher

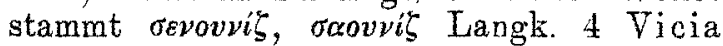
faba L.? [Sehweinsbohne]. Man könnte sehr leicht an das ägyptische $\bigcap_{\delta \odot}^{\text {me }} 0111$ śnnwt-t (Pap. Ebers LI 15), oder (Pap. Hearst III 4) denken. Im Aegyptischen heisst es von dieser Pflanze, dass sie "wächst auf ihrem Bauch wie die kid-t Pflanze usw." Diese Art des Wachsens passt auf eine kriechende oder kletternde Pflanze, was wiederum mit der Bestimmung des orvovi's als Vicia stimmt, obwohl eben bei $\nabla$. faba diese Eigenschaft nicht so ausgeprägt ist. Wenn es weiter in der Beschreibung der ägyptischen Pfl. heisst, dass sie "Blüte setzt wie sšn $\mathrm{Pfl}$. ", mit der Lotusblume de- terminiert, scheint dies dagegen zu sprechen; indessen wissen wir nicht bestimmt, was sšn ist (eine Art Lotus?), wie man sich auch denken könnte, dass die bekannte LotusFigur wegen ihrer entfernten Aehnlichkeit - wenigstens in der Schrift - mit einer Viciablïte mangels besseren Zeichens benutzt werden könnte. Uebrigens steht in Pap. Hearst nicht sšn, sondern śrd PAl.

\section{Zu dem demotiscien Ostrakon mit jüdischen Eigernamen.}

Von W. Spiegelberg.

In meiner Notiz über das demotische Ostrakon von Tell el Jehûdije im letzten Hefte dieser Zeitschrift (S. 595) vergass ich auf die Schwierigkeit hinzuweisen, welehe meine Datierung des demotischen Textes schafft. Denn wenn man das Ostrakon spätestens in die Zeit des Ptolemaios IV Philopator setzt ${ }^{1}$ ), so kann es mit dem erst unter Ptolemaios VI Philumetor (um 160 v. Chr.) gebauten Oniastempel nichts zu tun haben. Will man also nicht annehmen, dass das Stüek durch Zufall ${ }^{2}$ ) an die Stelle geraten ist, an der es gefunden wurde, also von weither dorthin verschleppt worden ist, so bleibt auch die Möglichkeit bestehen, es zu einer jüdischen Ansiedelung in Beziebung zu setzen, die vor dem Bau des Oniastempels hier bestauden hat. Denn dass dieser Tempel in einer schon bestehenden jüdischen Kolonie des Deltas gebaut wurde, ist a priori wahrscheinlich. Jedenfalls ist in Erwägung aller Umstände - auch abgesehen von der paläographischen Datierung ${ }^{3}$ ), die ich bei unserer gegenwärtigen Kenntnis der demotischen Schrift nicht mit absolnter Sicherheit zu geben wage - die Annahme sehr bedenklich, dass die in dem Ostrakon genannten Ziegelarbeiter an dem Oniastempel gebaut haben. W. Spiegelberg.

1) $\mathrm{Za}$ dieser Datierung scheint auch Griffith zu neigen. "Mr. Griffith agrees that it may be as late as Ptolemy Philometor, though he would have been inclined to date it rather earlier (Petrie in Text der in Frage stehenden Publikation $S$ 26).

*) Dafür könnte sprechen, dass in der ganzen grossen Anlage nur dieses eine Ostrakon zu Tage gekommen ist.

3) Sie ist mir allerdings sehr wahrscheinlich. 